\title{
THE RELATION BETWEEN THE FAST VITAL CAPACITY CURVES AND THE MAXIMUM BREATHING CAPACITY
}

\author{
BY \\ L. BERNSTEIN AND G. KAZANTZIS \\ From the Department of Physiology, The London Hospital Medical College, London, E.I
}

(RECEIVED FOR PUBLICATION JULY 19, 1954)

The maximum breathing capacity (M.B.C.) of an individual is defined as the greatest amount of air he can breathe in one minute. The test in which it is measured was introduced by Hermannsen (1933) as a test of the mechanical efficiency of the respiratory apparatus. Since then it has been widely accepted as fulfilling that purpose and has been quoted (Gray, Barnum, Matheson, and Spies, 1950) as the best single index of the maximum ventilatory capacity of an individual. Many workers have considered that the test is an exhausting one to perform, particularly for subjects with respiratory disfunction, and have, in consequence, attempted to replace it by one which in their view is less severe. The curve recorded by a spirometer, when an individual expires his whole vital capacity volume into it as rapidly as he is able, we have called the "expiratory fast vital capacity" (E.F.V.C.) record. It has also been termed (Kennedy, 1953) the "expiratory vital spirogram" (E.V.S.). Most of the attempts at finding a substitute for the M.B.C. test have been efforts to predict what should be the M.B.C. of the subject from measurements of the quantity of air breathed out in various fractions of time during an E.F.V.C. recording. Such attempts, using the whole of the vital capacity, have been made by Cournand, Richards, and Darling (1939) and also by Gilson and Hugh-Jones (1949); while Roche and Thivollet (1949), Tiffeneau, Bousser, and Drutel (1949), Kennedy (1950), Kadlec and Vyskocil (1950), Gaensler (1951), Guillet (1951), Hanaut (1953), and Kennedy (1953) have used the part of the vital capacity expired in some arbitrarily chosen time interval. The last of these papers contains an extensive review of the previous work in this field.

\section{Criticism of Existing Methods of Predicting M.B.C.}

Fig. 1 shows at the left an E.F.V.C. record made by a subject who then, without removing the breathing mask from his face, continued for a few breaths breathing as deeply as he was able at a respiratory rate of about 60 breaths a minute (middle record). The record at the extreme right is an "inspiratory fast vital capacity" (I.F.V.C.) record from the same individual. This was produced by the subject, having first expired as deeply as he could, inspiring his full vital capacity volume from the spirometer as rapidly as he could.

Fig. 2 has been traced from Fig. 1, but the various curves have been transposed horizontally in order that partial superimposition shall indicate the resemblances between certain portions of them. The expiratory phase of the maximal ventilation curve is very like a part of the E.F.V.C. record. This resemblance, which has been noted before (Kennedy, 1953), is the basis of most attempts at predicting M.B.C., or at substituting measurements made from the E.F.V.C. record for direct measurement of the M.B.C.

Generally, the method used has been to measure the volume of air expelled in some arbitrary fraction of time after beginning the E.F.V.C. record and to show that this correlates highly with the subject's M.B.C. There has been much disagreement as to what is the ideal time interval. Thus Tiffeneau and others (1949), Roche and Thivollet (1949), Gaensler (1951), and Hirdes and van Veen (1952) have used one second, while Kennedy (1953) has used 0.75 second and has criticized those workers using the longer time interval. All these workers have assumed that the division of time between the expiratory and inspiratory phases of maximum effort respiration is relatively constant, so that the arbitrarily chosen time interval corresponds to the expiratory phase of the maximum effort respiration. Thus Kennedy (1953) states that 0.75 second corresponds to a respiratory rate of 40 breaths per minute; the implied assumption is that, expiration and inspiration being of equal duration, a complete breath occupies 1.5 seconds (or one fortieth of a minute). Reference 


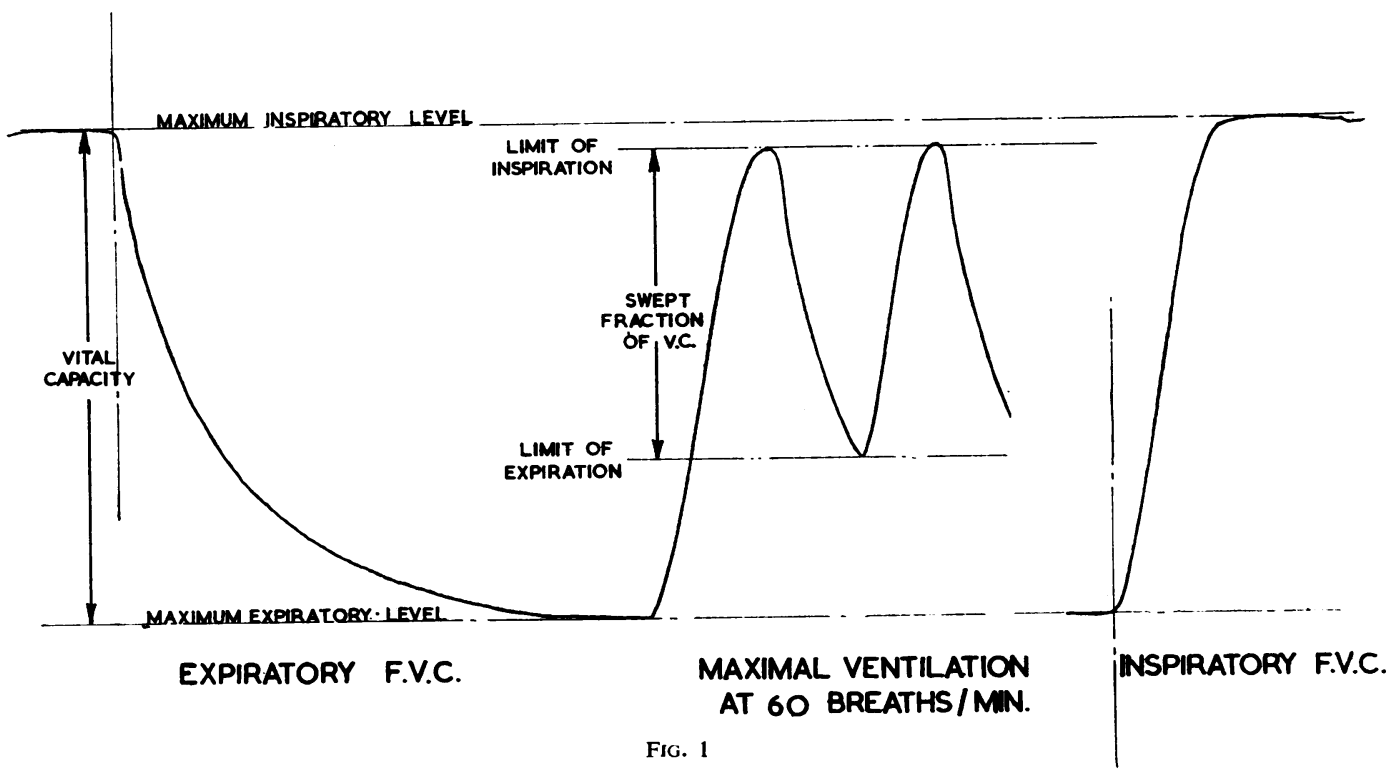

Fig. 1.-Expiratory F.V.C. curve, maximal tidal ventilation curve (at about 60 breaths/min.) and inspiratory F.V.C. curve recorded by the same individual. Between recording the E.F.V.C. curve and the tidal ventilation curve the individual did not remove the breathing mask from his face.

Fig. 2. - The curves of Fig. 1 have been traced, and have been partially superimposed so as to reveal the similarity between the expiratory phase of the maximal tidal ventilation curve and a portion of the E.F.V.C. curve, and between the inspiratory phase and a portion of the I.F.V.C. curve.

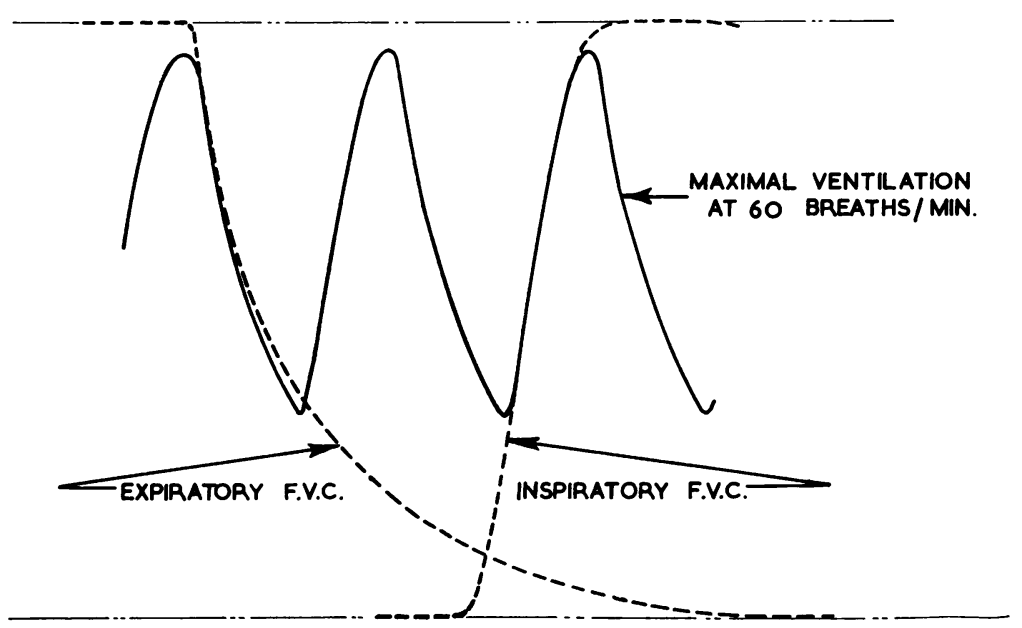

Fig. 2

to Fig. 1 or Fig. 2 shows that the time sharing between expiratory and inspiratory phases for the subject who produced this record of maximal ventilation is not equal, but is in the proportion expiration/inspiration $\simeq 58 / 42$. In a group of $\operatorname{six}$ maximal ventilation tracings, at two different respiratory rates, taken at random from our collected records, the time sharing between the two phases of respiration was as shown in Table I. The mean fraction of a cycle occupied by inspiration at $\mathbf{3 0}$ breaths/min. is statistically significantly different from that at 70 breaths $/ \mathrm{min}$. $(t=4.1$, d.f. $=5, P \simeq 0.01$ ). Clearly the duration of the inspiratory phase depends on the respiratory rate 
selected by the subject performing the maximal ventilation.

If expiration be assumed to occupy some fixed proportion of the time available for a breath, then the expiratory F.V.C. curve alone cannot justifiably be used for predicting the M.B.C., and, instead, some method of prediction should be employed in which use is made of both the inspiratory and the expiratory F.V.C. curves. Fig. 2 shows that the I.F.V.C. curve, in this subject, is at least as good a fit to the inspiratory phase of the maximal tidal ventilation curve as is the E.F.V.C. curve to the expiratory phase, and this finding constitutes yet another reason for employing both curves.

Another assumption is implied by using prediction methods in which the volume of air is measured over the first second (or some arbitrarily chosen fraction of a second) of the E.F.V.C. effort. This assumption is that, in maximal ventilation, expiration always begins from the position of full inspiration, and consequently that the first (and the fastest) part of the E.F.V.C. curve corresponds to the expiratory phase of maximum tidal ventilation. Cournand and others (1939) noted that the last part of the vital capacity is expired very slowly, and Kennedy (1953) proceeded from this to state that if this part is used the minute volume will not be maximal. A similar view was expressed by Hirdes and van Veen (1952). This view completely ignores the existence of the inspiratory process as a part of maximal ventilation. Fig. 2 shows that the last part of the vital capacity is also inspired very slowly. Thus what is gained by expiring over the first part of the E.F.V.C. curve may well be lost by inspiring over the last part of the I.F.V.C. curve. In Figs. 1 and 2, when breathing maximally at 60 breaths a minute, the subject concerned does not use the part of his vital capacity corresponding to full inspiration, and Bernstein, D'Silva, and Mendel (1952) have shown that in maximal effort breathing the level reached in inspiration (here referred to as the "limit of inspiration") gradually falls from about $98 \%$ of full inspiration at 20 breaths a minute to about $90 \%$ at 70 breaths a minute. Unless the limit of inspiration for a given individual performing the M.B.C. test is known in advance, it is not possible to decide what portion of his E.F.V.C. curve should be used for measuring the air expelled in any arbitrarily chosen time.

There is yet another criticism which may be levelled at most of the previous attempts at predicting M.B.C. from E.F.V.C. curves. This is the uncertainty which exists as to whether the observed values these workers have obtained for the M.B.C. in their subjects, and which are correlated with the predicted values in the process of validating the prediction method, really represent the maximum breathing capacity. This uncertainty arises because of the way in which the M.B.C. test is customarily performed. The usual method (Gilson and Hugh-Jones, 1949) is to instruct the subject, breathing into the recording spirometer, to breathe "as deep as you can, as fast as you can." The subject is then assumed in some unknown way to find the best combination of depth and rate of respiration, which affords the highest value for this minute ventilation. Now there is really no reason a priori to assume that this is the case. No experimental evidence supporting this notion has been adduced, and the measure obtained in this way is better referred to as the "maximum voluntary ventilation" (M.V.V.). Evidence to the contrary has been produced by D'Silva and Mendel (1950) and by Bernstein and others (1952), who showed that the maximum minute ventilation at a selected respiratory rate, which they have termed the "maximum ventilatory capacity" (M.V.C.), increases with the respiratory rate in normal subjects up to rates of about 70 to 80 breaths a minute. At higher rates the increase, if any, is insignificant. When the M.B.C. is determined in the conventional way, with selection of rate and depth by the subject, the rate selected is often about 40 breaths a minute. At this rate the maximum minute ventilation is usually considerably lower than that at 70 breaths a minute, and cannot therefore reasonably be regarded as representing the M.B.C. which, in consequence of the definition of the term, must be the highest value for the individual's minute ventilation. Probably the other workers have failed to notice that an increase in respiratory rate would have afforded an increase in the subject's minute ventilation (over and above the presumed M.B.C.) because conventional spirometers, such as they have used, possess large recording errors in the frequency range $45-80$ breaths a minute (Bernstein and Mendel, 1951), and this is just the range in which the increase in minute ventilation must be sought. The reason why individuals left to choose their own balance of rate and depth usually breathe at about 40 breaths a minute is not understood. Possibly, at this rate, an optimum ratio of work done to minute ventilation may exist. This is at least as likely as that the individual unconsciously selects the combination giving the highest minute ventilation.

Clearly, then, the methods of predicting M.B.C. which have been used hitherto are based on several 
misconceptions. A method should be found in which use is made of both inspiratory and expiratory F.V.C. curves, in which allowance is made for the variability (between subjects and at different respiratory rates) in the limit of inspiration chosen by the subject, and in which the validity of the method is demonstrated by correlating the predicted values not with the M.V.V. at the patient's own selected rate but instead with the M.V.C. at that particular respiratory rate for which the prediction is to be made.

\section{A Hypothesis Concerning the Relation} Between the F.V.C. Curves and the Maximum Ventilatory Capacity

Reference to Fig. 2 suggests that the spirographic record of the tidal excursion in maximal ventilation could perhaps be regarded as being composed of intersecting portions of the inspiratory and expiratory F.V.C. curves. This would be expected if the F.V.C. curves represent the greatest effort of which the subject is capable at all times during the expiratory or inspiratory process. If the subject performing maximal ventilation also exerts the greatest possible effort, the inspiratory and expiratory phases of the maximal ventilation record must be portions of the respective F.V.C. curves. If, during a short spell of maximal breathing, the limit of inspiration does not alter, the respiratory process will consist of an expiration which follows the E.F.V.C. curve until a point is reached from which it is just possible to return along the I.F.V.C. curve to the original limit of inspiration in the time available for one breath. This point we shall term the "limit of expiration." The volume between the limits of expiration and inspiration will be the maximum tidal volume which can be achieved in the time available for one breath at the selected respiratory rate. This volume, using the terminology of D'Silva, Freeland, and Kazantzis (1953), we shall call the "swept volume" for the chosen respiratory rate (Fig. 1). If the swept volume is expressed as a fraction of the vital capacity it is termed the "swept fraction" (S.F.). The swept fraction will depend on the limit of inspiration selected. Figs. 1 and 2 show that, if the limit of inspiration is raised, use will be made of a part of the E.F.V.C. curve where the slope is higher-i.e., where the flow rate is greater-while at the same time a portion of the I.F.V.C. curve will be used where the slope is lower-i.e., the flow rate is less. Thus the effect of raising the limit of inspiration will be to change the inspiratory and expiratory flow rates in opposite senses. Unless the increase in one is always exactly counterbalanced by the corresponding decrease in the other-and this, from the appearance of the curves, is most unlikely-there will be some value for the inspiratory limit which will result in an optimal combination of inspiratory and expiratory flow rates, i.e., one at which the greatest possible swept volume will be achieved in the time available for a single breath.

When subjects perform the maximum ventilatory capacity test at selected and controlled respiratory rates (Bernstein and others, 1952) they are exhorted to breathe as deeply as they can at the prescribed rate. It is possible that they may unconsciously choose that level for the limit of inspiration which gives the largest swept volume at the particular respiratory rate. If it could be shown that the theoretical optimal limit of inspiration and the theoretical maximal swept volume deduced from an analysis of the subject's inspiratory and expiratory F.V.C. curves, at any chosen respiratory rate, agreed closely with the observed values at the same rate, this would both support the hypothesis developed in the preceding paragraphs, and also lend support to the contention of D'Silva and Mendel (1950) and Bernstein and others (1952) that the performance of the M.V.C. test at controlled respiratory rates affords the maximum minute ventilation of which the subject is capable at each respiratory rate.

Accordingly, an attempt was made to predict from a study of the inspiratory and expiratory F.V.C. curves of a series of subjects the way in which the optimal limit of inspiration and the optimal swept volume were related to the respiratory rate.

\section{METHOD}

Six healthy male subjects were selected. They were all medical students or members of the staff of the department of physiology. Each performed the fastest possible expiration of his vital capacity volume into a recording spirometer of the type described by Bernstein and others (1952). Without removing the breathing mask from his face, he continued to breathe for a short period as deeply as he could at a prescribed respiratory rate, the rate being controlled by breathing in time to a metronome. When a sufficiently long record had been obtained, usually 10 to 15 breaths long, the subject was asked to expire as deeply as he could and then to inspire his whole vital capacity volume as rapidly as he could. During the whole procedure the subject remained connected to the spirometer, so that the maximal ventilation record when compared with the F.V.C. records gave an accurate indication of the limit of inspiration chosen by him. The whole procedure was performed by each subject at respiratory rates between 20 and 
100 breaths a minute. From the records, the limit of inspiration and the swept volume at each respiratory rate were then measured.

In order to obtain the corresponding predicted values a somewhat complex procedure was necessary. For each subject transparencies were made of representative E.F.V.C. and I.F.V.C. curves by tracing each on to a sheet of celluloid. Over each curve a series of horizontal scaling lines was also traced so as to indicate the levels corresponding to various percen- precise registration of the zero and $100 \%$ levels) until the points on the two curves corresponding to $94 \%$ of full inspiration were separated by a horizontal distance representing a time interval of 1.2 seconds (equal to the duration of one breath at 50 breaths a minute). This horizontal distance was calculated from the known time calibration of the original F.V.C. records. The volume at which the two F.V.C. records intersected represented the limit of expiration. Subtracting this from the limit of inspiration gave the

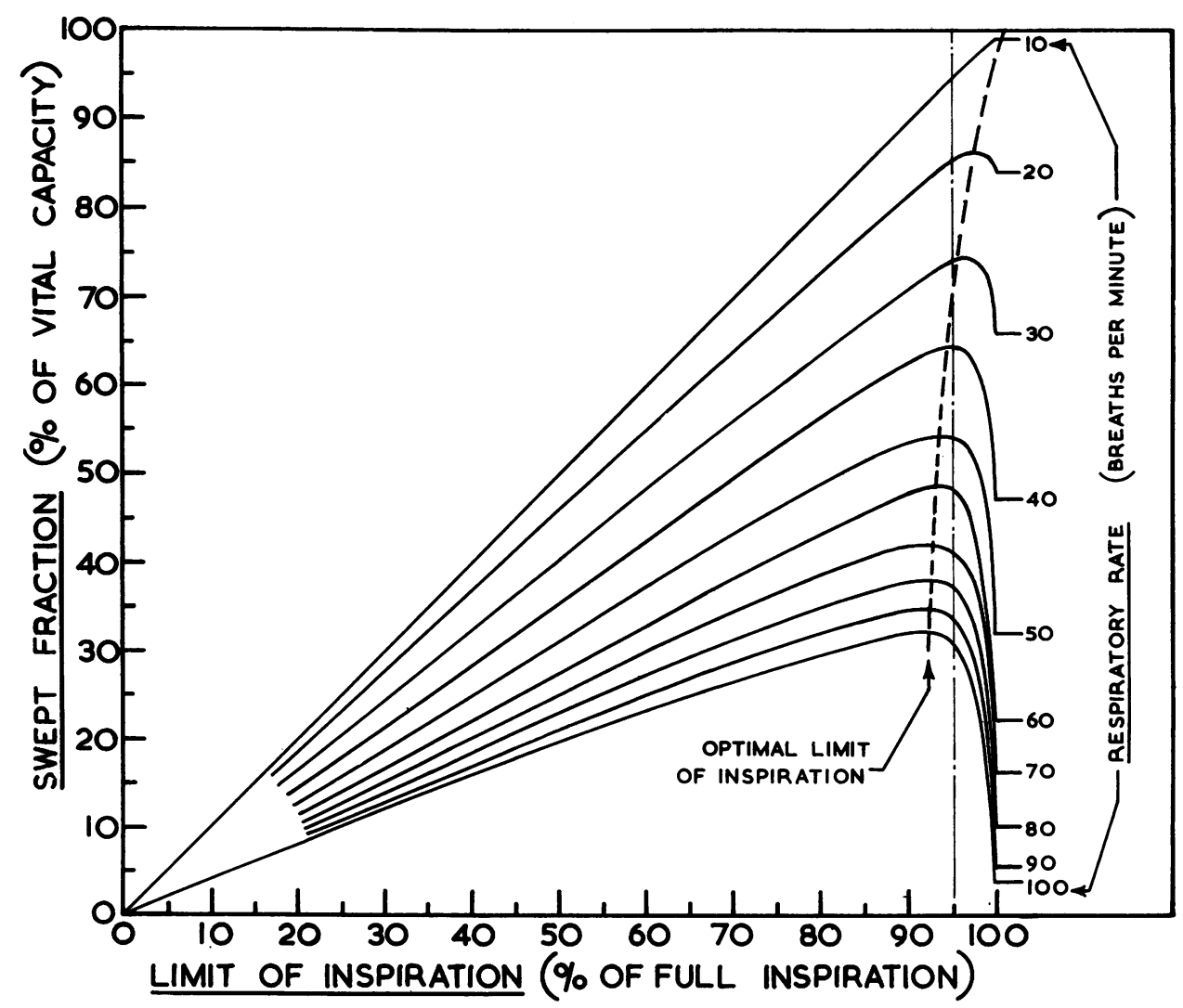

Fig. 3.-Family of curves deduced from the F.V.C. curves of Fig. 1, and relating the theoretical sivept fraction to arbitrarily selected limits of inspiration at various respiratory rates for the subject who recorded the F.V.C. curves.

tages of full inspiration. Between the position of full expiration and $90 \%$ of full inspiration these lines were drawn at $10 \%$ intervals. Between 90 and $100 \%$ of full inspiration they were drawn at $2 \%$ intervals. The I.F.V.C. transparency was now superimposed on the E.F.V.C. transparency, displaced to the right, and was adjusted vertically so that the zero and $100 \%$ scaling lines on the two transparencies were in exact registration. If, for example, the swept volume corresponding to a limit of inspiration at $94 \%$ of full inspiration and a respiratory rate of 50 breaths a minute was to be predicted, the I.F.V.C. transparency was moved horizontally (still maintaining the predicted swept fraction corresponding to the chosen limit of inspiration and the chosen respiratory rate.

For each of the following respiratory rates $(10,20$, $30 \ldots 100$ breaths a minute) the theoretical swept fraction corresponding to each of the following limits of inspiration $(100,98,96 \ldots 90,80,70 \ldots 10 \%$ of full inspiration) was predicted-a total of 140 predicted values for each subject. From the results obtained, a family of curves was plotted for each subject, an example for one subject being shown by the continuous line curves in Fig. 3. This example is quite typical of the others obtained. 


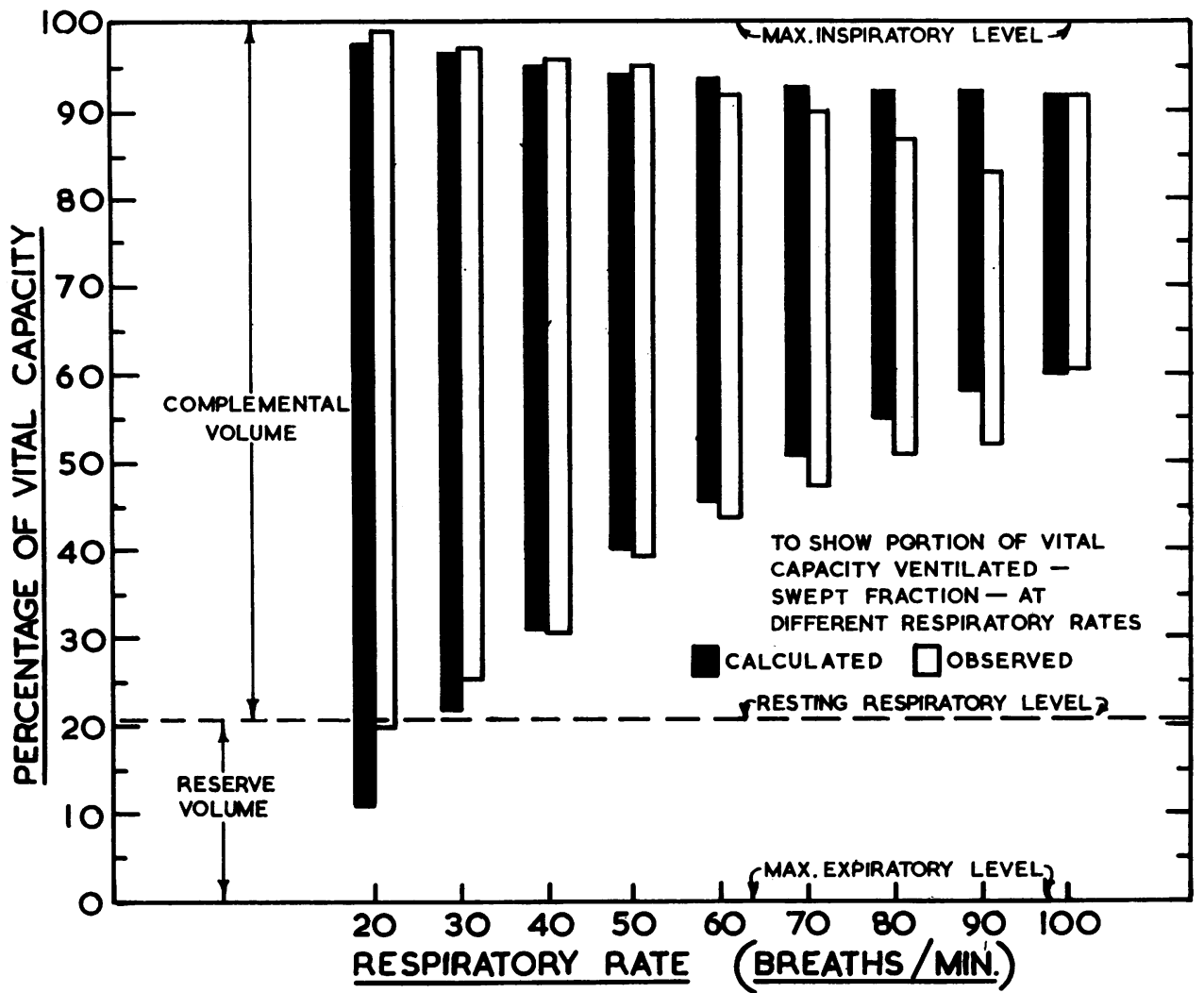

FIG. 4. - Predicted and observed values for the swept fraction and for the limit of inspiration at various respiratory rates in the subject to whom Figs. 1 to 3 apply.

\section{RESULTS}

Fig. 3 shows that for any chosen respiratory rate there is one value for the limit of inspiration at which the swept fraction is a maximum. At 10 breaths a minute this optimal inspiratory limit is $100 \%$-i.e., full inspiration. As the respiratory rate increases the optimal limit of inspiration falls, until at 100 breaths a minute it is only $92 \%$ of full inspiration. The dashed line in Fig. 3 roughly illustrates this trend, but should not be regarded as an accurate indication of the optimal limits of inspiration at the various respiratory rates. These findings are in agreement with the hypothesis advanced in earlier paragraphs concerning the relation between the F.V.C. curves and the swept fraction in maximal ventilation.

Fig. 4 shows the predicted values for the optimal limit of inspiration and the optimal swept fraction at each respiratory rate (black columns) compared with the corresponding observed values (white columns). These data are for the same subject as Fig. 3. Except in two instances (80 and 90 breaths a minute) the agreement is good, and even at these rates the agreement is good for the swept fractions: it is in the values for the limits of inspiration that the greater discrepancies occur. Results of the same general form were obtained for each of the other subjects studied.

Fig. 5 is a scatter diagram illustrating the agreement between the predicted and observed swept fractions at all respiratory rates from 20 to 100 breaths a minute in all six subjects. The correlation coefficient between the predicted and observed values is $r=+0.972$ and the regression equation for the observed values on the predicted values is

$$
\text { S.F.OBS }=0.94 \text { S.F.PRED }+1.35
$$

Fig. 6 is drawn to the same conventions as Fig. 4 , but shows the mean results for the six subjects investigated. Once more the close resemblance between the predicted and observed values is evident. In this diagram the open circles represent the mean results obtained for the limit of inspiration and the swept fraction of a group of 14 normal young subjects investigated by Bernstein 


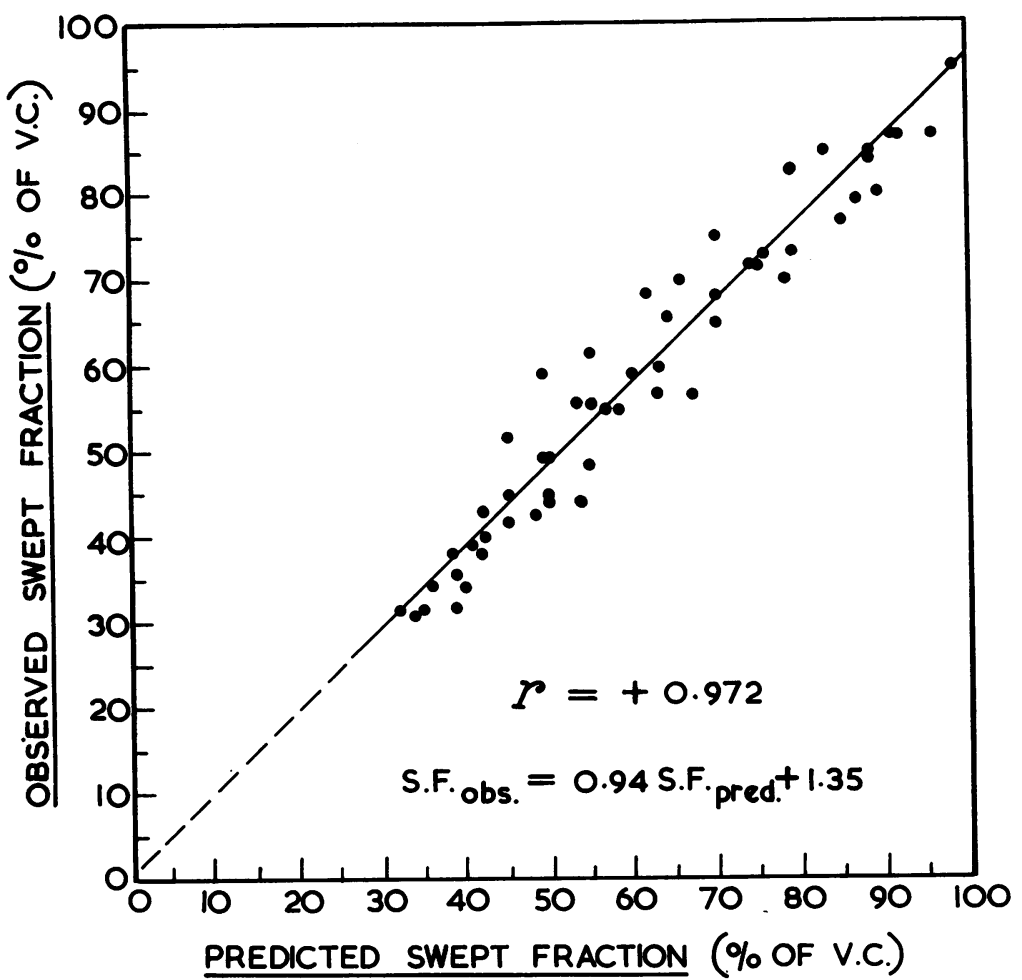

Fig. 5
FIG. 5.-Scatter diagram for the predicted and observed values of the swept fraction at various respiratory rates in a group of six subjects. The line is the regression line for observed swept fraction on predicted swept fraction.

FIG. 6.-The vertical columns represent the mean predicted and observed swept fraction at each of nine different respiratory rates, and the corresponding mean predicted and observed optimal limits of inspiration, 'for the same group of six subjects as are represented in the data of Fig. 5. The open circles and the dashed line represent the corresponding data for the group of 14 subjects investigated by Bernstein and others (1952).

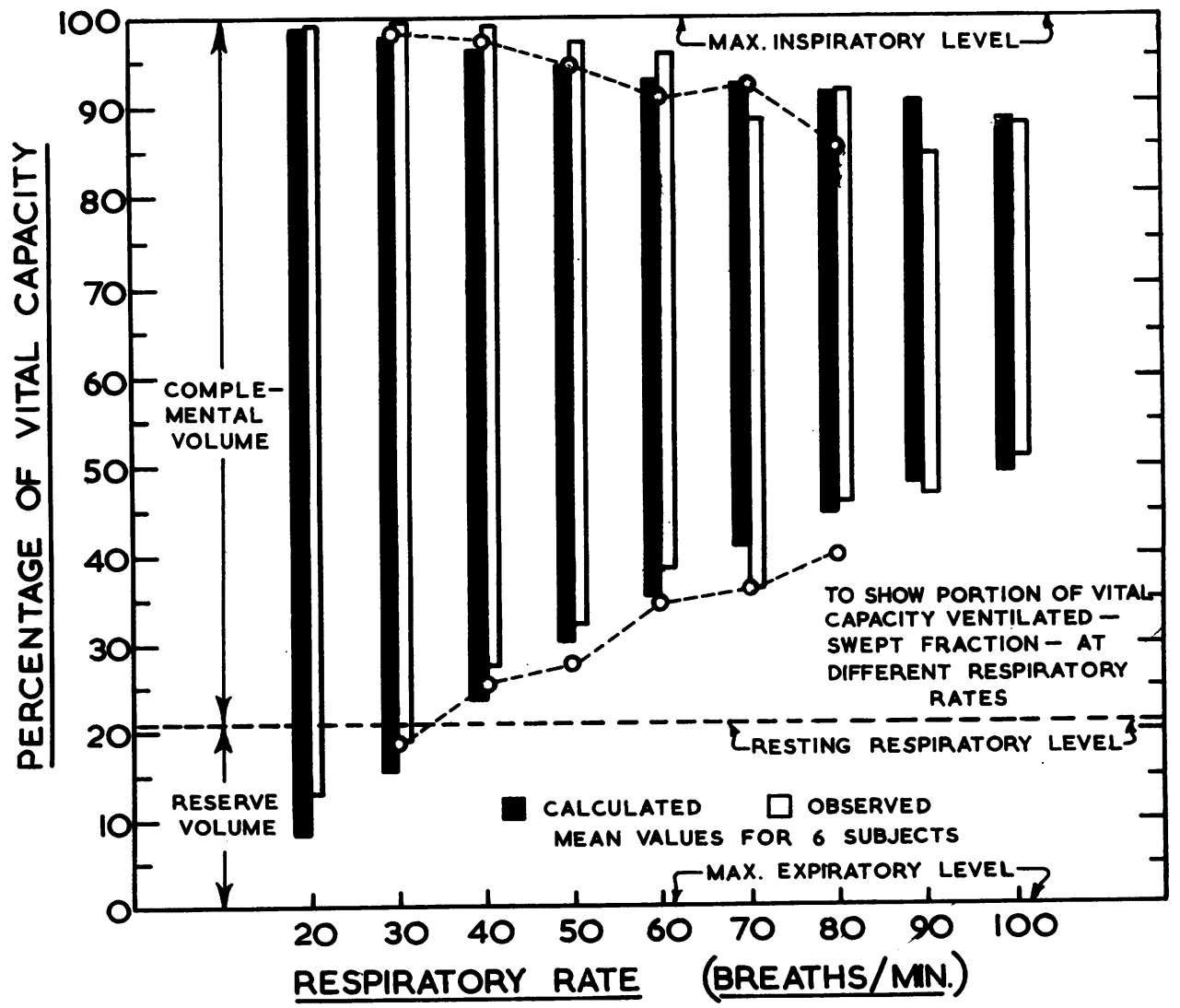

FIG. 6 
and others (1952), and are taken from their records. Clearly, our predicted and observed results bear a close resemblance to the results obtained by these authors. These latter results refer to an entirely unassociated group of subjects. The data were obtained more than 12 months before the formulation of the hypothesis which gave rise to the present investigation, and the observers were different in the two instances.

\section{Discussion}

These results amply justify the hypothesis which was earlier advanced, namely, that the curve of maximum tidal ventilation may be regarded as being composed of intersecting portions of the expiratory and inspiratory F.V.C. curves, these portions being so related as to afford the greatest possible swept volume in the time available for a single breath. In addition, the finding of Bernstein and others (1952), that the limit of inspiration in maximum ventilation tends to fall as the respiratory rate rises, is confirmed both by direct observation and also by the agreement between observation and prediction from the hypothesis. The curves shown in Fig. 3 assist us to understand the basis of this phenomenon. The poorer agreement between the predicted and observed limits of inspiration than between the predicted and observed swept fractions is also explicable in terms of Fig. 3, whence it can be seen that the value of the predicted optimal swept fraction at a given respiratory rate is but slightly subject to uncertainty, because, except at the rate of 10 breaths a minute, the curves are very flat in the region of their maximum. This very fact accounts for the greater uncertainty in deciding what are the limits of inspiration at which these optimal swept fractions are achieved, and this uncertainty is reflected in the poorer agreement between the predicted and observed values for the limits of inspiration.

From the predicted and observed swept fractions, the corresponding predicted and observed M.V.C.s for each respiratory rate can be calculated as the product of the swept fraction and the particular respiratory rate. Since the swept fraction is itself the swept volume expressed as a percentage of the vital capacity, this procedure yields the M.V.C. not in absolute units, i.e., litres per minute, but relative to the subject's vital capacity, i.e., as $\mathrm{x}$ times the vital capacity per minute. For the purpose of comparing the performance of a single individual at various respiratory rates this procedure makes no difference to the results. For the simultaneous comparison of a number of subjects at various respiratory rates the procedure in no way distorts the effect of respiratory rate on M.V.C. but it reduces the range of variation of the individual measurements at any one respiratory rate, and so renders easier the graphical representation of the results. This process has been performed and the data obtained are shown in Fig. 7. The black circles represent the mean predicted M.V.C. at each respiratory rate, and the vertical lines drawn through them indicate the range of the six separate values from which each mean was calculated. The white circles indicate the corresponding mean observed M.V.C.s and the lines again indicate the ranges of the separate observations. The extraordinarily good agreement between the predicted and observed values is, of course, merely a reflexion of the pre-existing agreement between the predicted and observed swept fractions: in effect each value represented in Fig. 7 is derived from a value in Fig. 6 multiplied by a constant, the respiratory rate. But the figure also shows that the M.V.C. theoretically should rise with increasing respiratory rate up to rates of about 80 breaths a minute. The white squares, and the lines drawn through them, in Fig. 7 are corresponding mean observed M.V.C. values for the 14 subjects used by Bernstein and others (1952). These show that our predicted and observed values - the latter obtained by a different observer on a totally different group of subjects -agree very closely with their observed values, confirming that their contention, that the M.V.C. rises with increasing respiratory rate up to rates of 70 or 80 breaths a minute, is a well-justified one. This being so, the M.V.V., which is usually determined at or about 40 breaths a minute, cannot represent the subject's M.B.C.- whatever else it may represent. As D'Silva and Mendel (1950) and Bernstein and others (1952) have stated, the M.B.C. can only be estimated by measuring a subject's M.V.C. over a full range of respiratory rates until the curve of M.V.C. plotted against rate reaches a maximum. This maximum M.V.C. is then the best estimate obtainable of the subject's M.B.C.

All the criticisms of existing methods of predicting M.B.C., which were made earlier in this paper, have been justified by the present experimental analysis of the form of the tidal ventilation curve in maximal ventilation. However, the success achieved by these methods by Kennedy (1953), who has obtained a correlation coefficient of $r=$ +0.927 between the measurement of slope of a portion of a subject's F.V.C. curve and that subject's own M.V.V., suggests that in practice they 


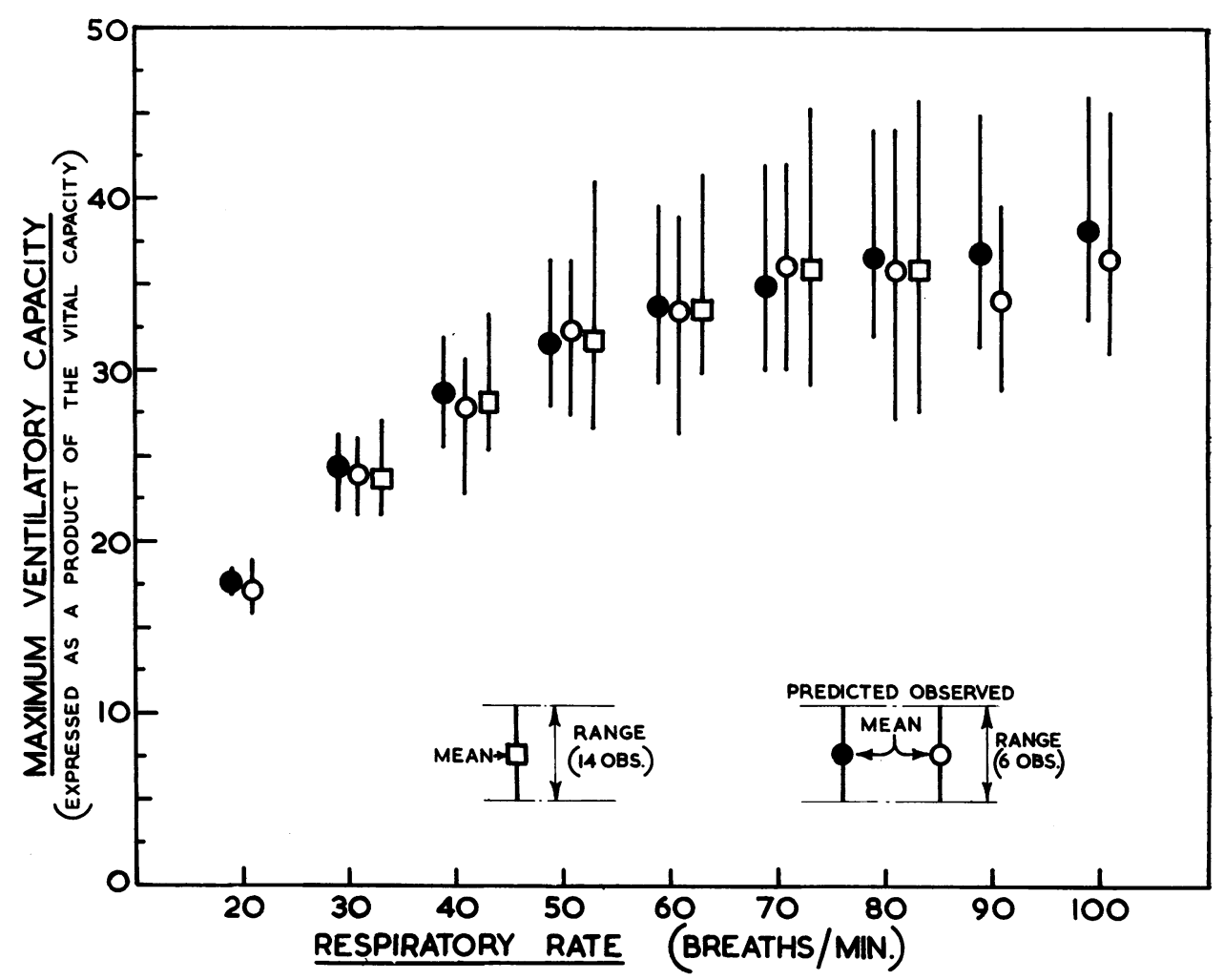

Fig. 7.-Mean (and range) of predicted and observed M.V.C.s at various respiratory rates for the group of six subjects represented in Figs. 5 and 6 . The corresponding mean values (and ranges) for the 14 subjects of Bernstein and others (1952) are also shown.

are reasonably reliable. The success of these supposedly invalid methods must seem to vitiate the hypothesis on which they are deemed invalid. If it could be shown that this success were itself predictable from the hypothesis, it would then become further evidence in support of the hypothesis.

Kazantzis (1953) and D'Silva and Kazantzis (1954) have shown that, while the E.F.V.C. curves of individuals are different one from another, they can, by suitable scaling of the coordinates on which they are drawn, all be reduced to a common form, in which they can be superimposed on one another with considerable accuracy. The curve which they regard as the typical E.F.V.C. curve is a hyperbola, which is smoothly concave upwards. It may be objected that this form of curve is different from those reported elsewhere in the literature (Kennedy, 1950, 1953; Kadlec and Vyskocil, 1950; Kennedy and Stock, 1952 ; Hirdes and van Veen, 1952). All except the last of these authors have described curves which appear to contain a periodic component, i.e., which appear to pass through several maxima and minima. However,
Bernstein (1954) has shown that these curves are probably the result of periodic recording artefacts, caused by resonance phenomena in the spirometers used by these authors. He has shown that curves similar to those described by these authors can be synthesized by adding the known recording error of a particular spirometer to a smooth curve of the kind described by D'Silva and Kazantzis (1954). The synthetic curve is then in very close agreement with that actually recorded on the defective spirometer. Hirdes and van Veen have recorded curves similar to those of D'Silva and Kazantzis, and have also realized the importance of spirographic artefacts, but they have mistakenly regarded their own records as consisting of rectilinear and curvilinear portions intersecting at a critical point. It is therefore reasonable to accept D'Silva and Kazantzis' curve as the basis for further discussion.

Fig. 8 shows four typical E.F.V.C. curves of the kind recorded by D'Silva and Kazantzis. Curve $A$ is that of an individual with an average value of time constant ( 0.5 second) and an average vital capacity (4.5 litres). Curve $B$ is that of an individual having twice as long a time constant and 
the same vital capacity. Curves $C$ and $D$ are for individuals with time constants of 0.5 second and 1.0 second, respectively, but with vital capacities of only 2.25 litres. At time $t_{1}$ an ordinate has been drawn intersecting the four curves at the points marked $a_{1}, b_{1}, c_{1}, d_{1}$, respectively. At time $t_{2}$ a second ordinate has been erected intersecting the curves at the points marked $a_{2}, b_{2}, c_{2}, d_{2}$, respectively. The volumes at which the $t_{1}$ ordinate intersect the four curves have the relative order of magnitude indicated by $a_{1}>b_{1}>c_{1}>d_{1}$, and this order is the same as the relative order of magnitude of the volumes at which the $t_{2}$ ordinate intersects them, i.e., $a_{2}>b_{2}>c_{2}>d_{2}$. It may be shown mathematically (or empirically, by drawing various combinations of curves for hypothetical individuals with different time constants and vital capacities) that this property, of constant relative order of magnitude for the intercepts made by ordinates at different times, remains true except in certain extreme instances where the vital capacity and the time constant are both grossly reduced.

There are many ways in which the correlation between two variables may be measured. The measure most commonly employed is the product moment correlation coefficient, $r$. This is a measure of the correlation between the observed values of the two variables. A second, somewhat similar, measure is the rank correlation coefficient, $r_{s}$, of

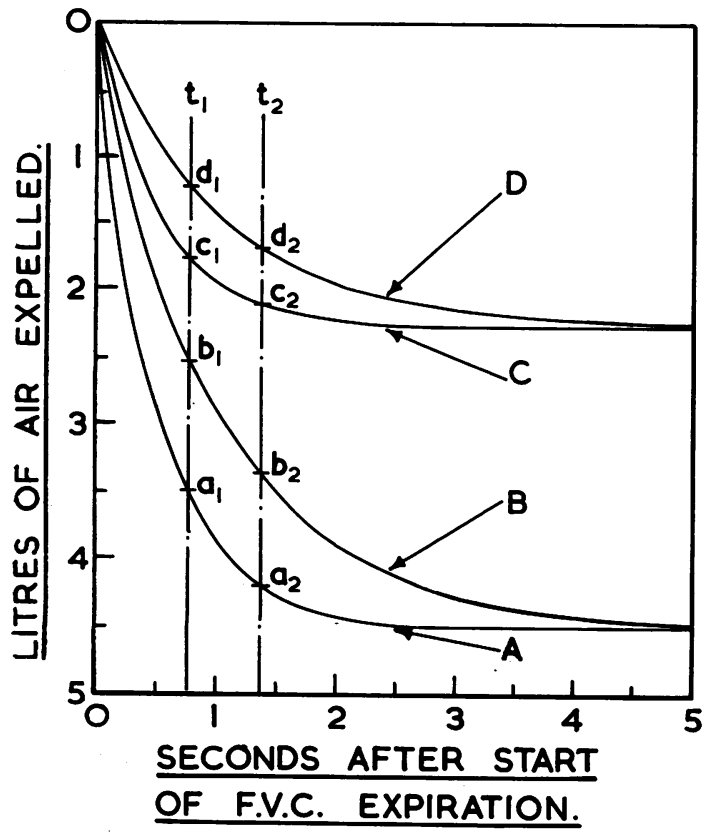

FIG. 8.-Diagram to illustrate certain properties of a family of E.F.V.C. curves.
Spearman. Here, instead of correlating the observed values themselves, it is their respective rank orders of magnitude which are correlated. The precise relationships which exist between the two correlation coefficients are not known. It is known, however, that when there is no correlation both methods yield the same correlation coefficient, i.e., $r=r_{s}=0$, and similarly when the correlation between the variables is perfect both methods yield the same correlation coefficient, i.e., $r=$ $r_{s}= \pm 1$. For degrees of correlation between tnese extremes, the two coefficients do not usually differ by more than about 0.02 (Guilford, 1950; Snedecor, 1946).

As has been shown, the volume expired in the first $t_{1}$ seconds of a set of E.F.V.C. curves will in general have the same rank order of magnitude as the volumes expired in the first $t_{2}$ seconds. It follows that the rank correlation coefficient for the $t_{1}$ volumes with the subjects' own M.V.C.s will have about the same numerical value as the rank correlation coefficient for the $t_{2}$ volumes and the M.V.C.s. But since each of these rank correlation coefficients is nearly equal to the corresponding product moment correlation coefficient, it also follows that the two product moment coefficients will differ by only a small amount.

If this argument is valid the correlation coefficient between M.V.C., or M.V.V., and the volume breathed out in the first $t$ seconds of an E.F.V.C. curve might be expected to have a nearly constant value, irrespective of the time interval, $t$, chosen, provided that the same time interval is chosen for all the subjects concerned. By determining whether this is actually so the validity of the argument which has led to this conclusion may be tested. The data of D'Silva and Kazantzis (1954) provided the material for such a test. These authors measured, in a group of 14 subjects, the correlation between M.V.C. at a stated respiratory rate and the volume of air expelled in the first $t$ seconds of an E.F.V.C. effort, where $t$ was one half of the duration of a single breath at the chosen rate-assuming that this period corresponded to the expiratory phase of maximal tidal ventilation. They did this at three respiratory rates, 30,50 , and 70 breaths a minute, the corresponding time interval being 1.0 seconds for 30 breaths a minute, 0.6 seconds for 50 breaths a minute, and 0.43 seconds for 70 breaths a minute. Using their data, what might be termed the "incorrect" correlation coefficients have been calculated, i.e., between the M.V.C. at 30 breaths a minute and the volumes expelled in the first 0.6 seconds and in the first 0.43 seconds, also between 
the M.V.C. at 50 breaths a minute and the volumes expelled in each of the other "incorrect" time intervals, and so also at 70 breaths a minute.

Table II shows the results obtained. The first column gives the respiratory rates for which the M.V.C.s were measured. The second gives the time intervals for which the volumes of air expired

\section{TABLE II}

CORRELATION BETWEEN M.V.C. AT STATED RESPIRATORY RATES AND VOLUME OF AIR EXPELLED IN DIFFERENT FRACTIONS OF AN F.V.C. EXPIRATION

\begin{tabular}{|c|c|c|c|c|}
\hline $\begin{array}{c}1 \\
\text { Respiratory } \\
\text { Rate at } \\
\text { which } \\
\text { M.V.C. was } \\
\text { Measured } \\
\text { (Breaths/ } \\
\text { min.) }\end{array}$ & $\begin{array}{l}2 \\
\text { Time over } \\
\text { which } \\
\text { Expired } \\
\text { Volume was } \\
\text { Measured } \\
\text { (sec.) }\end{array}$ & $\begin{array}{c}\text { 3 } \\
\text { Correlation } \\
\text { Coefficient }\end{array}$ & $\begin{array}{c}4 \\
\begin{array}{c}\text { Difference } \\
\text { between } \\
\text { " Correct } \\
\text { and }\end{array} \\
\text { " Incorrect } " \\
\begin{array}{c}\text { Values of } \\
r\end{array}\end{array}$ & $\begin{array}{c}5 \\
\text { Probability } \\
\text { that the } \\
\text { Value in } \\
\text { Column } 4 \\
\text { is Due to } \\
\text { Chance } \\
\text { (P) }\end{array}$ \\
\hline 30 & $\begin{array}{l}1.00 \\
0.60 \\
0.43\end{array}$ & $\begin{array}{l}0.930 \\
0.889 \\
0.840\end{array}$ & $\begin{array}{l}\overline{\overline{0}} \\
-0.041 \\
-0.090\end{array}$ & $\begin{array}{l}0.9>\overline{\mathbf{P}}>0.8 \\
0.4>\mathbf{P}>0.3\end{array}$ \\
\hline 50 & $\begin{array}{l}1.00 \\
0.60 \\
0.43\end{array}$ & $\begin{array}{l}0911 \\
0890 \\
0.858\end{array}$ & $\begin{array}{l}+0.021 \\
-0.032\end{array}$ & $\begin{array}{l}0.8>\mathrm{P}>0.7 \\
0.8>\bar{P}>0.7\end{array}$ \\
\hline 70 & $\begin{array}{l}1.00 \\
0.60 \\
0.43\end{array}$ & $\begin{array}{l}0.805 \\
0.780 \\
0.750\end{array}$ & $\begin{array}{c}+0.055 \\
+0.030 \\
-\end{array}$ & $\begin{array}{c}0.9>P>0.8 \\
0.9>P>0.8 \\
-\end{array}$ \\
\hline
\end{tabular}

For all comparisons $n_{1}=n_{2}=14$, d.f. $=11$.

were measured. The times corresponding to half the duration of a single breath at each respiratory rate are shown in heavy type. The third column shows the relevant correlation coefficients, with the "correct" coefficient at each respiratory rate shown in heavy type, so as to distinguish it from the "incorrect" ones. The differences between the "correct" and the "incorrect" coefficients are shown in column 4. These differences were tested for statistical significance by the use of Hotelling's (1940) test. The results are shown in column 5 of the table. The values here represent the probability, $P$, that a difference as large as, or larger than, that given on the corresponding line in column 4 could be occasioned by chance. None of the differences is significant by the accepted criteria of statistical significance. It may be accepted that if the M.V.C.s of a series of individuals, at some selected respiratory rate, are correlated with the volumes expired by those individuals in some chosen time interval after the start of their F.V.C. expirations, the value of the correlation coefficient will be independent of the time interval selected, provided that the same time interval is used for all individuals.

The M.B.C. of an individual could be predicted from his E.F.V.C. curve alone if the duration of the expiratory phase of his maximal tidal ventilation curve, at the respiratory rate corresponding to his highest M.V.C., were precisely known, and if the corresponding limit of inspiration were also known. The duration of the expiratory phase depends on two factors: the shape of the I.F.V.C. curve, and the optimal respiratory rate. When the respiratory rate is high the intersection of the two F.V.C. curves to form the maximum tidal ventilation curve occurs high up on the E.F.V.C. curve, where its slope is high, and where small differences in the slope of the inspiratory I.F.V.C. curve will have a considerable effect on the volume at which the intersection occurs, i.e., the swept volume. It might therefore be expected in these instances that if the I.F.V.C. curve is disregarded the predicted swept volumes should be subject to considerable uncertainty. On the other hand, when the respiratory rate is low the intersection occurs low down on the E.F.V.C. curve, where the slope is low, and where a small difference in the slope of the I.F.V.C. curve will have relatively little effect on the volume at which the intersection occurs, i.e., the swept volume. In this instance, it might be expected that the swept volumes predicted from the E.F.V.C. curve alone should have a much greater reliability. If this argument is valid, the correlation between the M.V.C. at a chosen respiratory rate and the volume of air expelled in some chosen time interval after the start of an F.V.C. expiration, while being independent of the time interval chosen, should depend on the respiratory rate chosen, so that at low respiratory rates better correlations should be obtained than at high rates. Reference to Table II will show that this is exactly what is found to occur. If, for each of the three time intervals, the three correlation coefficients corresponding to the three respiratory rates are compared, it will be seen that, of the nine possible comparisons of correlation coefficients, seven indicate a fall in the correlation as the respiratory rate rises, one indicates no appreciable change, and in only one instance (the correlations for 30 and for 50 breaths a minute with the time interval 0.43 seconds) is there an increase in the correlation with increasing respiratory rate. Individually, none of the nine possible comparisons reveals a statistically significant difference-even the most highly significant only affords a value for $P \simeq 0.02$ - but the steady trend toward smaller correlation coefficients with rising respiratory rate suggests that it is a real effect rather than a chance finding. (If, by chance, an increase of correlation were as likely as a decrease in any one comparison of coefficients, then the probability that seven out of nine comparisons would yield decreases and only one would yield an increase-as is here the case-would be $0.02>\mathrm{P}>0.002$.) 
From the preceding arguments it would then appear that it should be possible to predict with accuracy the M.V.C. at any low respiratory rate from a measurement of the volume of air expelled in some stated time after the start of an F.V.C. expiration, so that, provided the same time interval is used for all the subjects, the predicted and observed M.V.C.s will correlate highly irrespective of what time interval is chosen.

Various workers (Bernstein, and others, 1952 ; Kennedy, 1953) have shown that when subjects perform the M.B.C. test under the conditions prescribed by Gilson and Hugh-Jones (1949) they commonly breathe at about 40 to 50 breaths a minute. The resulting measure, which is really the M.V.V. and not the M.B.C., will lie very close in value to the subject's own M.V.C. at about 45 breaths a minute. Thus, when this measure is correlated with the volume of air expelled in some fraction of time after the start of a F.V.C. expiration, the correlation is found to be a fairly high one, irrespective of the time interval selected. This explains why the workers using such different time intervals as 0.75 seconds, or 1.0 seconds, or " one-half the duration of a single breath at $x$ breaths a minute," have obtained equally good correlations, and why even a (theoretically) infinite time interval, as is the case when the whole of the vital capacity is used as the volume to be correlated with the M.V.V., yields a correlation coefficient as high as $r=+0.826$ (Gilson and Hugh-Jones, 1949). It must, however, be appreciated that these workers have not demonstrated a correlation between their various measures and the M.B.C., but instead with the subject's M.V.C. at a rate of about 45 breaths a minute. The conclusion must be drawn that the whole of the controversy as to what is the correct portion of the E.F.V.C. curve to use for prediction purposes is, by the very nature of the problem, sterile.

It might now be pertinent to enquire, "If these admittedly theoretically invalid techniques give such good results in practice, what purpose is served by the long analysis of their invalidity and by their subsequent rehabilitation?" The answer, we feel, is in the following argument. To accept a prediction method as reliable, on the evidence of its success as revealed by a high correlation between predicted and observed values, but without any hypothetical basis for expecting the results to be so well correlated, is to commit the statistical sin of confusing a correlation with a causal relationship. As long as predictions are confined to subjects and situations which obviously differ but slightly from those used in testing the prediction method, there may be reasonable grounds for expecting similar relations to hold between the predicted and observed values in these subjects and situations. But as soon as the prediction technique is extended to other classes of subjects or to other conditions, e.g., a change of respiratory rate, an unjustifiable extrapolation is made. For example, it has here been shown that the prediction of M.V.C. from the E.F.V.C. curve alone is quite reliable at low respiratory rates, but its reliability decreases when it is applied to high respiratory rates. By investigating the theoretical basis of the prediction method a better understanding of the relation between the F.V.C. curves and the M.V.C. has been secured, a technique for predicting M.V.C. which is reliable over a wide range of respiratory rates has been discovered, and the dangers of using any such technique in conditions for which its use has not been validated have been made more apparent. In addition certain facts, which hitherto were merely isolated findings and which were rather in dispute, have been explained-such as the change in limit of inspiration with change of respiratory rate, or the rise in M.V.C. with increase of respiratory rate up to about 70 breaths a minute -and because a theoretical basis for these findings has been provided they have been confirmed to an extent which otherwise would have required very many further observations.

It is pertinent to comment further on the assumption implied in a prediction method employing only the E.F.V.C. curve. As has already been suggested, if the I.F.V.C. curve could be disregarded without affecting the reliability of the prediction this could only be because there was an invariable relationship between the shape of the two F.V.C. curves. Now it is not known whether or not this is so. It would be wrong either to accept or deny it without experimental or observational test. If it is so accepted, the opportunity of gathering valuable information may be lost: it is quite possible that the way in which the curves vary in relation to each other may be of equally great, or even greater, significance for diagnostic purposes than the variation in either curve alone-it is not possible to say. But if the importance of the relations between the two curves is once realized the necessary information may be sought.

It may also be questioned whether or not it is wise to regard the study of the F.V.C. curves merely as a substitute for the M.B.C. test. Enquiry must first be made as to the purpose served by the M.B.C. test. Since there is very little knowledge of what are the actual physiological mechanisms 
which limit the ventilatory efficiency of an individual, nor any knowledge of how to measure them directly, it is necessary to rely, for purposes of diagnosis, differential diagnosis, or prognosis, on empirically demonstrated relationships, statistically significant correlations or associations, between performance level in some test of ventilatory efficiency and one or other disease state. The whole value of the M.B.C. test in this context is that the subject is presumed to be stressed to the very limit in the performance of this particular physiological function. Now, there are, of course, many different ways in which the ventilatory function might be limited. The limitation could be physical: simply that above a certain rate of air flow the resistance to flow, or to tissue deformation, increases so steeply that no further increase of flow is possible. It could be physiological: for example, the ratio between work done and rate of flow might be optimal at some particular rate of flow, or the ratio between expulsive pressure and rate of flow might be optimal at some particular rate of flow, or there might simply be a limit to the expulsive and impulsive forces available. Or, again, the limitations might be psychological: some individuals just not being willing to exert as much effort as others. So long as the M.B.C. test is performed by different methods by different observers, i.e., by some observers allowing free choice of rate and depth (M.V.V.) and by others setting chosen respiratory rates (M.V.C.), it is not at all certain that they are all measuring the same limiting factors. $A$ priori the M.V.V. method seems to be more likely to give information about psychological limitations or about optimal ratios or work to flow rates, whereas the M.V.C. method seems more likely to provide information about limiting deformation rates, or flow rates, or about limiting pressures. It is not suggested that there is, theoretically, any reason to suppose that either method of performing the test gives more valuable information than does the other, but they probably give different information, and until the way in which performances in the two kinds of test are related to each other, or to disease states, is understood it is presumptuous to regard either as the correct way of performing the M.B.C. test. However, once it has been demonstrated that subjects in performing the M.V.C. test may exceed their own performances in the so-called M.B.C. test (in reality the M.V.V. test) the term M.B.C., used in this context, clearly has become misleading. It is therefore suggested that the use of the term M.B.C. should be discontinued and that the two terms M.V.V. and
M.V.C., as they have been defined in this paper, should be used instead.

Secondly, it might be considered whether either the M.V.V. or the M.V.C. test offers as much information as could be obtained by a study of the E.F.V.C. and I.F.V.C. curves. Now, in demonstrating the relations between these curves and the M.V.C. it has been shown that the M.V.C., at any chosen respiratory rate, can be predicted from the two F.V.C. curves. If the prediction were perfect, i.e., if the correlation coefficient between predicted and observed values was $r= \pm 1$, the information provided by the F.V.C. curves would be equivalent to that provided by the M.V.C. In fact, the information about maximum ventilation given by the F.V.C. curves is less than that given by a direct M.V.C. test, because the correlation is not perfect. But it should be remembered that the overall measure of maximum ventilation is providing information about two processes, inspiration and expiration. It has here been demonstrated that both processes are concerned in the limitation of tidal volume at a given respiratory rate. If only the maximum tidal volume at any respiratory rate (i.e., the swept volume) is measured the opportunity is lost to enquire whether only one of the two processes, inspiration or expiration, is abnormal in character or whether both are abnormal. That is to say, information about the individual processes is lost. It is easy to see that a slowing of either the E.F.V.C. curve or the I.F.V.C. curve, or of both curves, could lead to identical reductions in the swept volume at some particular respiratory rate. This leads once more to a point made in an earlier paragraph: that the study of the interrelationships of the F.V.C. curves might therefore be much more rewarding than the present use of the E.F.V.C. curve merely as a substitute for the M.B.C. test.

\section{SUMmary}

Existing methods of predicting maximum breathing capacity (M.B.C.) from the expiratory fast vital capacity (E.F.V.C.) curve alone have been criticized.

A hypothesis has been presented: that the tidal ventilation curve in maximum effort breathing is composed of optimally related portions of the E.F.V.C. and inspiratory fast vital capacity (I.F.V.C.) curves. This hypothesis has been confirmed experimentally.

A method has been developed for predicting maximum ventilatory capacity (M.V.C.) at any chosen respiratory rate, from measurements made on both E.F.V.C. and I.F.V.C. curves. It has been 
shown that predicted and observed values correlate highly over the range of respiratory rates from 20 to 100 breaths a minute, $r=+0.972$.

Certain earlier, and disputed, observations, that the limit of inspiration falls with increasing respiratory rate, and that M.V.C. rises with increasing respiratory rate up to rates of about 70 to 80 breaths a minute, have been confirmed by direct observation and have also been shown to be deducible from the hypothesis concerning the relation of the F.V.C. curves and the tidal ventilation curve.

The high correlations, between predicted and observed maximum voluntary ventilation (M.V.V.) values, obtained by earlier workers using theoretically unsound prediction techniques based on the E.F.V.C. curve alone, have been shown to be deducible from the hypothesis. The range within which these techniques may be applied has been shown to be limited.

The relations between the various measures has been discussed, and the suggestion has been made that a study of the F.V.C. curves for their own sakes might be more rewarding than to regard them merely as substitutes for the M.B.C. test.

It has been suggested that because the M.B.C. has become confused with the M.V.V. and because, when the term is so used it does not accord with its own definition, the use of the term should be discontinued, the two methods of testing ventilatory efficiency being referred to as M.V.V. (where rate and depth are freely chosen by the subject) and M.V.C. (where rate is specified and controlled by the observer).

\section{REFERENCES}

Bernstein, L. (1954). Thorax, 9, 63

D'Silva, J. L., and Mendel, D. (1952). Ibid., 7, 255.

- and Mendel, D. (1951). Ibid., 6, 297.

Cournand, A., Richards, D. W., and Darling, R. C. (1939). Amer. Rev. Tuberc. 40, 487.

D'Silva, J. L., Freeland, D. E., and Kazantzis, G. (1953). Thorax, 8, 303.

and Kazantzis, G. (1954). Ibid., 9, 128

- and Mendel, D. (1950). Ibid., 5, 325 .

Gaensler, E. A. (1951). Amer. Rev. Tuberc., 64, 256

Gilson, J. C., and Hugh-Jones, P. (1949). Clin. Sci., 7, 185

Gray, J. S., Barnum, D. R., Matheson, H. W., and Spies, S. N. (1950). J. clin. Invest., 29, 677.

Guilford, J. P. (1950). Fundamental Statistics in Psychology and Education, 2nd ed., p. 313. McGraw-Hill, London, New York. Guillet, M. (1951). La Place de la Spirographie dans l'Expertise en Matiere de Silicose. Imprimerie Grandville, Nancy.

Hanaut, A. (1953). Proc. 10th Int. Congr. on Industr. Med., 1951, Lisbon (cited by Kennedy, M. C. S. (1953) in Thorax, 8, 73).

Hermannsen, J. (1933). Z. ges. exp. Med., 90, 130.

Hirdes, J. J., and Veen, G. van (1952). Acta tuberc. scand., 26, 264.

Hotelling, H. (1940). Ann. math. Statist., 11, 271.

Kadlec, K., and Vyskocil, J. (1950). Pracovni Lekarstvi, 6, 348. (cited by Kennedy, M. C. S. (1953) in Thorax, 8, 73).

Kazantzis, G. (1953). J. Phrsiol., Lond., 122, 77P.

Kennedy, M. C. S. (1950). Beitr. Silikose-Forsch., Heft 10, 19.

Kennedy, 1953$)$. Thorax, 8, 73 .

and Stock, J. P. P. (1952). Ibid., 7, 43

Roche, L., and Thivollet, J. (1949). Arch. Mal. prof., 10, 448.

Snedecor, G. W. (1946). Statistical Methods, 4th ed., p. 165. Iowa State College Press, Ames, Iowa.

Tiffeneau. R., Bousser, J., and Drutel, P. (1949). Paris méd. (partie méd.), 39, 543 . 